\title{
A Gravitational Wave Background from Primordial Black Hole Lattices in Matter Dominated Era
}

\author{
Ke Wang * \\ National Astronomical Observatories, \\ Chinese Academy of Sciences, \\ $20 A$ Datun Road, Beijing 100012, China
}

(Dated: January 23, 2019)

\begin{abstract}
We use the wide-used Einstein Toolkit to solve the Einstein constraints and then simulate the expansion of primordial black hole lattices (PBHLs) with different value of $f_{\mathrm{PBH}}$ and $m_{\mathrm{PBH}}$. We find that $f_{\mathrm{PBH}}$ plays an important role during the evolution of PBHLs. Since the motion of primordial black holes (PBHs) caused by the expansion of PBHLs occurs at speeds close to that of light, we expect the emission of gravitational waves (GWs) during the expansion of PBHLs. We use both analytical estimates and numerical simulations to cross check the production of GWs in expanding PBHLs.
\end{abstract}

\section{INTRODUCTION}

Observations of gravitational waves (GWs) by LIGO/VIRGO [1 5] have proved there should be a gravitational wave background (GWB) produced by the black hole and neutron star binaries' coalescence. Meanwhile, people also believe there is a primordial inflationary GWB from tensor perturbations generated by quantum fluctuations during inflation even though Planck didn't measure it 6]. Besides that, there are many other theories (or sources) which can produce GWBs, such as reheating after inflation [7, thermal phase transitions from the decays of cosmic strings [8]. Here we expect that there is a GWB from primordial black hole lattices (PBHLs) in matter dominated era.

To what extend can the configuration of primordial black holes (PBHs) just after the epoch of matter-radiation equality be considered as a PBHL? As we known, a pair of PBHs would decouple from the expansion of the Universe and form a gravitationally bound system if the average energy density of PBHs over the volume $R^{3}$ is larger than the total background cosmic energy density $\rho_{t}$, that is, if $m_{\mathrm{PBH}} R^{-3}>\rho_{t}$. Since $\rho_{t} \approx m_{\mathrm{PBH}} \bar{R}^{-3} f_{\mathrm{PBH}}^{-1}$, we know that most of PBHs are gravitationally free on average in matter dominated era. On the other hand, due to the cosmological principle that the Universe is homogeneous and isotropic on large scales, we can say that the PBHL is the most reasonable configuration of $\mathrm{PBHs}$ with a monochromatic mass distribution on our hands.

In fact, there is a cosmological model, black hole lattices (BHLs) 9 12, to investigate the so-called "backreaction" that the local inhomogeneities can affect the global expansion of the Universe. For review of BHLs, see [13]. It's worth pointing out that the PBHLs doesn't serve as a cosmological model like BHLs does but is an early epoch of the Universe. In practice, however, we do apply the same technologies developed by [10 12] when they evolve the BHLs in numerical relativity to the PHBLs.

Here we will turn to the wide-used Einstein Toolkit [14] to solve the Einstein constraints and simulate the spacetime and hydrodynamical evolution. More precisely: the thorn CT_MultiLevel (and CT_Analytic) [15] gets the initial data of PBHLs; the thorn McLachlan [16 18] evolves spacetime using the Baumgarte-Shapiro-Shibata-Nakamura (BSSN) formalism [19 21]; the thorn GRHydro evolves the hydrodynamical system [22 24].

This paper is organized as follows. In Subsec. IIA, we give the Einstein constraints of PBHLs with dust. In Subsec. IIB, we solve the Einstein constraints of PBHLs with different value of $f_{\mathrm{PBH}}$ and $m_{\mathrm{PBH}}$. In Subsec. IIIA, we show the expansion of PBHLs with different value of $f_{\mathrm{PBH}}$ and $m_{\mathrm{PBH}}$. In Subsec. IIIB we use both analytical estimates and numerical simulations to derive the production of GWB in PBHLs. At last, a brief summary and discussion are included in Sec. IV]

In this paper, we adopt the following conventions: Greek indices run in $\{0,1,2,3\}$, Latin indices run in $\{1,2,3\}$ and repeated indices implies summation and we are in a geometric unit system with $G=c=M_{\odot}=1$.

* wangke@itp.ac.cn 


\section{INITIAL DATA OF PRIMORDIAL BLACK HOLE LATTICES}

To mock an infinite 3-dimensional lattice in our simulations, we impose the periodic boundary conditions on a cubic with $x^{i}$ in $[-5,5]$. A PBH is located at the center of cubic and surrounded by flat-distributed dust with energy density $\rho_{m}$. It's worth noting that our simulation is not done in Schwarzschild coordinates but in its isotropic coordinates. For simplicity, we just add the dust outside the PBH horizon $r_{h}=0.5 m_{\mathrm{PBH}}$ so that the inner boundary conditions of PBHLs can be set as that of a single puncture [25]. And there are no outer boundary conditions for PBHLs. Although the set up of initial data is usually a non-trivial task, here we can solve PBHLs' coupled system of one non-linear and three linear elliptic partial differential equations thanks to [10, 12, 15].

\section{A. Einstein Constraints of Primordial Black Hole Lattices}

The initial data of PBHLs must satisfy their Hamiltonian constraint and momentum constraint

$$
\begin{aligned}
R+K^{2}-K_{i j} K^{i j} & =16 \pi E, \\
D_{j} K_{i}^{j}-D_{i} K & =8 \pi p_{i},
\end{aligned}
$$

where the 3-metric $\gamma_{i j}$ is the intrinsic metric, $K_{i j}$ is the extrinsic curvature, $R$ is 3 -Ricci scalar, $D_{i}$ is the covariant derivative associated with $\gamma_{i j}, E$ is the matter energy density and $p_{i}$ is the matter momentum density as measured by the Eulerian observer. After conformal decomposition of $\gamma_{i j}$ and $K_{i j}$ with the conformal factor $\Psi$

$$
\begin{aligned}
K_{i j} & =A_{i j}+\frac{1}{3} K \gamma_{i j} \\
\gamma_{i j} & =\Psi^{4} \tilde{\gamma}_{i j} \\
A_{i j} & =\Psi^{-10} \hat{A}_{i j}
\end{aligned}
$$

we can get the conformal Einstein constraints

$$
\begin{aligned}
\tilde{D}_{i} \tilde{D}^{i} \Psi-\frac{1}{8} \tilde{R} \Psi+\frac{1}{8} \hat{A}_{i j} \hat{A}^{i j} \Psi^{-7}+2 \pi E \Psi^{5}-\frac{1}{12} K^{2} \Psi^{5} & =0 \\
\tilde{D}_{j} \hat{A}^{i j}-\frac{2}{3} \Psi^{6} \tilde{D}^{i} K & =8 \pi \Psi^{10} p^{i}
\end{aligned}
$$

where $\tilde{D}_{i}$ is the covariant derivative associated with the conformal metric $\tilde{\gamma}_{i j}$. Adopting the conformal transverse traceless method

$$
\begin{aligned}
\hat{A}^{i j} & =\tilde{D}^{i} X^{j}+\tilde{D}^{j} X^{i}-\frac{2}{3} \tilde{D}_{k} X^{k} \tilde{\gamma}^{i j}+\hat{A}_{\mathrm{TT}}^{i j} \\
\tilde{D}_{j} \tilde{A}^{i j} & =\tilde{D}_{j} \tilde{D}^{j} X^{i}+\frac{1}{3} \tilde{D}^{i} \tilde{D}_{j} X^{j}+\tilde{R}_{j}^{i} X^{j}
\end{aligned}
$$

and choosing the free data as following

$$
\begin{aligned}
\tilde{\gamma}^{i j} & =\delta^{i j} \\
\hat{A}_{\mathrm{TT}}^{i j} & =0 \\
E & =\Gamma^{2}\left(\rho_{m}+P_{m}\right)-P_{m}=\rho_{m} \\
p^{i} & =\left(E+P_{m}\right) v_{m}^{i}=0 \\
K & =K_{c} W(r)=K_{c}\left\{\begin{array}{l}
0, \quad 0 \leq r \leq l \\
\left(\frac{(r-l-\sigma)^{6}}{\sigma^{6}}-1\right)^{6}, \quad l<r<l+\sigma \\
1, \quad l+\sigma \leq r \leq L,
\end{array}\right.
\end{aligned}
$$

we can get the conformal transverse traceless form of Einstein constraints for PBHLs

$$
\begin{aligned}
\triangle \psi+\triangle\left(\frac{m_{\mathrm{PBH}}}{2 r} W(r)\right)-\frac{1}{12} K^{2} \Psi^{5}+\frac{1}{8} \hat{A}_{i j} \hat{A}^{i j} \Psi^{-7}+2 \pi \rho_{m} \Psi^{5} & =0, \\
\triangle X^{i}+\frac{1}{3} \partial^{i} \partial_{j} X^{j}-\frac{2}{3} \Psi^{6} \partial^{i} K & =0,
\end{aligned}
$$


where we have expanded the conformal factor as

$$
\Psi=\psi+\frac{m_{\mathrm{PBH}}}{2 r}(1-W(r))
$$

according to the inner boundary conditions of a single puncture.

\section{B. Solving the Einstein Constraints}

We use a multigrid approach [15] to solving the Einstein constraints (6). But we impose the integrability condition as 10

$$
2 \pi m_{\mathrm{PBH}}-\frac{1}{12} K_{c}^{2} \int W^{2} \Psi^{5}+\frac{1}{8} \int \hat{A}_{i j} \hat{A}^{i j} \Psi^{-7}+2 \pi \int \rho_{m} \Psi^{5}=0,
$$

at the end of each relaxation step to determine the negative parameter $K_{c}$. Finally we perform the inner resetting

$$
\begin{aligned}
\psi(0) & =1, \\
X^{i}(0) & =0
\end{aligned}
$$

at the end of every relaxation step.

Tab. I shows the parameters setting of ten PBHLs and Tab. II shows the solution of the Einstein constraints of ten PBHLs when all of 5 refinement levels cover the whole domain with spacing $1,0.5,0.25,0.125$, and 0.0625 respectively. Comparing L0 to L7, L8 and L9, we can see that a smaller PBH mass $m_{\mathrm{PBH}}$ gives a shorter initial proper cubic edge $D_{\text {edge }}(\tau=0)$ and leads a lower initial expansion rate $-K_{c}$. Comparing L0 to L4, L5 and L6, we can see that a higher matter energy density $\rho_{m}$ has a higher initial expansion rate $-K_{c}$ but produces a shorter initial proper cubic edge $D_{\text {edge }}(\tau=0)$. Comparing L0 to L1, L2 and L3, we can see that a larger PBH mass $m_{\mathrm{PBH}}$ and a smaller matter energy density $\rho_{m}$ still produce a larger initial proper cubic edge $D_{\text {edge }}(\tau=0)$ but lead a lower initial expansion rate $-K_{c}$. That is to say, the initial expansion rate $-K_{c}$ is more sensitive to the matter energy density $\rho_{m}$ than the PBH mass $m_{\mathrm{PBH}}$. From the Tab. II] we can also see that a smaller PBH mass $m_{\mathrm{PBH}}$ violates the Hamiltonian constraint more severely.

\begin{tabular}{cccccccc}
\hline \hline Lattices & $f_{\mathrm{PBH}}$ & $m_{\mathrm{PBH}}$ & $\rho_{m}$ & $\rho_{t}$ & $L$ & $\sigma$ & $l=\frac{\mathrm{PBH}}{2}$ \\
\hline L0 & $100 \%$ & 2 & 0 & 0.002 & 5 & 3.5 & 1 \\
\hline L1 & $75 \%$ & 1.5 & 0.0005 & 0.002 & 5 & 3.75 & 0.75 \\
L2 & $50 \%$ & 1 & 0.001 & 0.002 & 5 & 4 & 0.5 \\
L3 & $25 \%$ & 0.5 & 0.0015 & 0.002 & 5 & 4.25 & 0.25 \\
\hline L4 & $80 \%$ & 2 & 0.0005 & 0.0025 & 5 & 3.5 & 1 \\
L5 & $66.7 \%$ & 2 & 0.001 & 0.003 & 5 & 3.5 & 1 \\
L6 & $62.5 \%$ & 2 & 0.0015 & 0.0035 & 5 & 3.5 & 1 \\
\hline L7 & $100 \%$ & 1.5 & 0 & 0.0015 & 5 & 3.75 & 0.75 \\
L8 & $100 \%$ & 1 & 0 & 0.001 & 5 & 4 & 0.5 \\
L9 & $100 \%$ & 0.5 & 0 & 0.0005 & 5 & 4.25 & 0.25 \\
\hline \hline
\end{tabular}

TABLE I: The parameters setting of ten PBHLs.

In Fig. 1, the $\Psi=\psi>1$ at the boundary means that each PBH is gravitationally coupled to its neighbors; the boundary $\psi$ of L0 is larger than that of L9 just because the PBH with larger mass will be gravitationally coupled to its neighbors more tightly; the boundary $\psi$ of L0 is larger than that of L6, which means the matter surrounding the PBH suppresses the gravitational correlation among PBHs; the boundary $\psi$ of L0 is larger than that of L3, which is consistent with above two cases. In Fig. 2, we give the solutions of $X_{1}$ of PBHLs. In Fig. 3, we shows the initial Hamiltonian constraint violation of PBHLs.

The last plot in Fig. 1. Fig. 2 and Fig. 3 is a convergence test for solving the initial data and shows the $\psi, X_{1}$ and $H$ of $\mathrm{L} 0$ with spacing of the finest one of 5 refinement levels equal to $0.0625\left(\mathrm{~L}_{f}\right), 0.078125\left(\mathrm{~L} 0_{m}\right)$ and 0.1041667 $\left(\mathrm{LO}_{c}\right)$ respectively. 


\begin{tabular}{ccccccc}
\hline \hline Lattices & $f_{\mathrm{PBH}}$ & $m_{\mathrm{PBH}}$ & $K_{c}$ & $D_{\text {edge }}(\tau=0)$ & $H_{\max }$ & $H_{\min }$ \\
\hline L0 & $100 \%$ & 2 & -0.2480 & 14.16 & $0.0023-0.0009$ \\
\hline L1 & $75 \%$ & 1.5 & -0.3413 & 12.03 & $0.0032-0.0021$ \\
L2 & $50 \%$ & 1 & -0.3864 & 10.76 & $0.0168-0.0114$ \\
L3 & $25 \%$ & 0.5 & -0.4000 & 10.07 & $0.2438-0.2868$ \\
\hline L4 & $80 \%$ & 2 & -0.3711 & 12.63 & $0.0047-0.0021$ \\
L5 & $66.7 \%$ & 2 & -0.4869 & 11.21 & $0.0087-0.0041$ \\
L6 & $62.5 \%$ & 2 & -0.6200 & 9.84 & $0.0178-0.0060$ \\
\hline L7 & $100 \%$ & 1.5 & -0.2338 & 13.12 & $0.0029-0.0020$ \\
L8 & $100 \%$ & 1 & -0.2120 & 12.07 & $0.0168-0.0114$ \\
L9 & $100 \%$ & 0.5 & -0.1698 & 11.01 & $0.2436-0.2868$ \\
\hline \hline
\end{tabular}

TABLE II: The solution of the Einstein constraints of ten PBHLs with spacing of the finest one of 5 refinement levels equal to 0.0625

\section{TIME EVOLUTION OF PRIMORDIAL BLACK HOLE LATTICES}

Given the initial data of PBHLs, we can simulate the time evolution of PBHLs. Due to the negative trace of the extrinsic curvature $K_{i j}$ around the boundary, we can expect an expansion of PBHLs during the time evolution. Meanwhile, since the motion of PBHs caused by the expansion of PBHLs occurs at speeds close to that of light, we also expect the emission of GWs during the time evolution.

\section{A. Expansion of Primordial Black Hole Lattices}

In order to circumvent the central puncture, here we will study the expansion of PBHLs through the rescaling of the proper length of lattice cells' edge with proper time. The proper time at anywhere is given by integrating the corresponding lapse function $\alpha(t, x)$ from the beginning of simulation

$$
\tau(t, x)=\int_{0}^{t} \alpha\left(t^{\prime}, x\right) d t^{\prime}
$$

where the evolution of $\alpha$ is given by

$$
\partial_{t} \alpha-\beta^{i} \partial_{i} \alpha=-2 K \alpha
$$

And the proper length measured in a cctk_delta_time (or a timestep) is then given by

$$
D(\tau)=\int_{\gamma_{\tau}}\left[\left(-\alpha^{2}(\tau, \ell)+\beta^{2}(\tau, \ell)\right)\left(\partial_{\ell} t\right)^{2}+2 \beta_{i}(\tau, \ell) \partial_{\ell} t \partial_{\ell} x^{i}+\gamma_{i j}(\tau, \ell) \partial_{\ell} x^{i} \partial_{\ell} x^{j}\right]^{1 / 2} d \ell,
$$

where $\beta_{i}(\tau, x)$ is the shift vector obeying a Gamma driver and $\gamma_{\tau}$ is a constant- $\tau$ edge parameterized by $\ell$.

Fig. 4 shows the expansion of four PBHLs with the smallest initial Hamiltonian constraint violation in each group, where a prime represents a derivative with respect to the proper time $\tau$. We can see that $L 0$ and $L 1$ evolve differently even though they share a same $\rho_{t}$. That is to say $f_{\mathrm{PBH}}$ will play an important role during the evolution of PBHLs. Comparing $L 4$ to $L 0$ and $L 1$, we find that the PBHL with a larger $\rho_{t}$ no matter due to an extra $\rho_{m}$ or a larger $m_{\mathrm{PBH}}$ expands faster. There is no intersection point between the evolutions of $D_{\text {edge }}(\tau)^{\prime}$, which means a PBHL with a larger $-K_{c}$ will keep expanding faster for ever. Finally, we also find that the motion of PBHs caused by the expansion of PBHLs does occur at speeds close to that of light.

The right plot in Fig. 4 shows the expansion of $L 0$ and Fig. 5 shows the $L_{2}$ norms of the Hamiltonian and momentum constraint for $L 0$ with spacing of the finest one of 5 refinement levels equal to $0.0625,0.078125$ and 0.1041667 respectively. They serve as a convergence test for evolving PBHLs. 

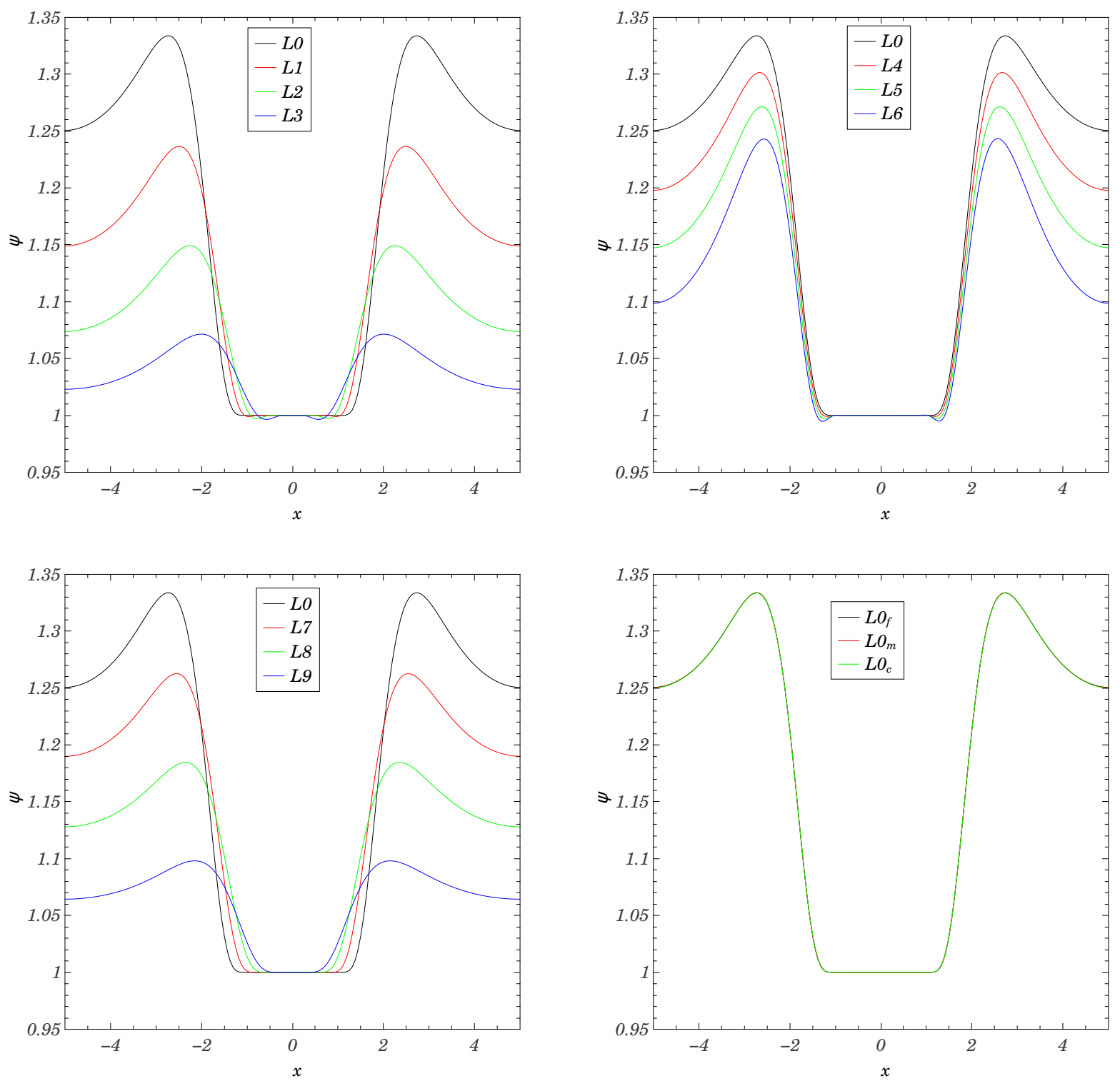

FIG. 1: $\psi$ of PBHLs as a function of position.

\section{B. Gravitational Waves in Primordial Black Hole Lattices}

We have shown that the motion of PBHs caused by the expansion of PBHLs occurs at speeds close to that of light. Here we will use both analytical estimates and numerical simulations to cross check the production of GWs in expanding PBHLs. 

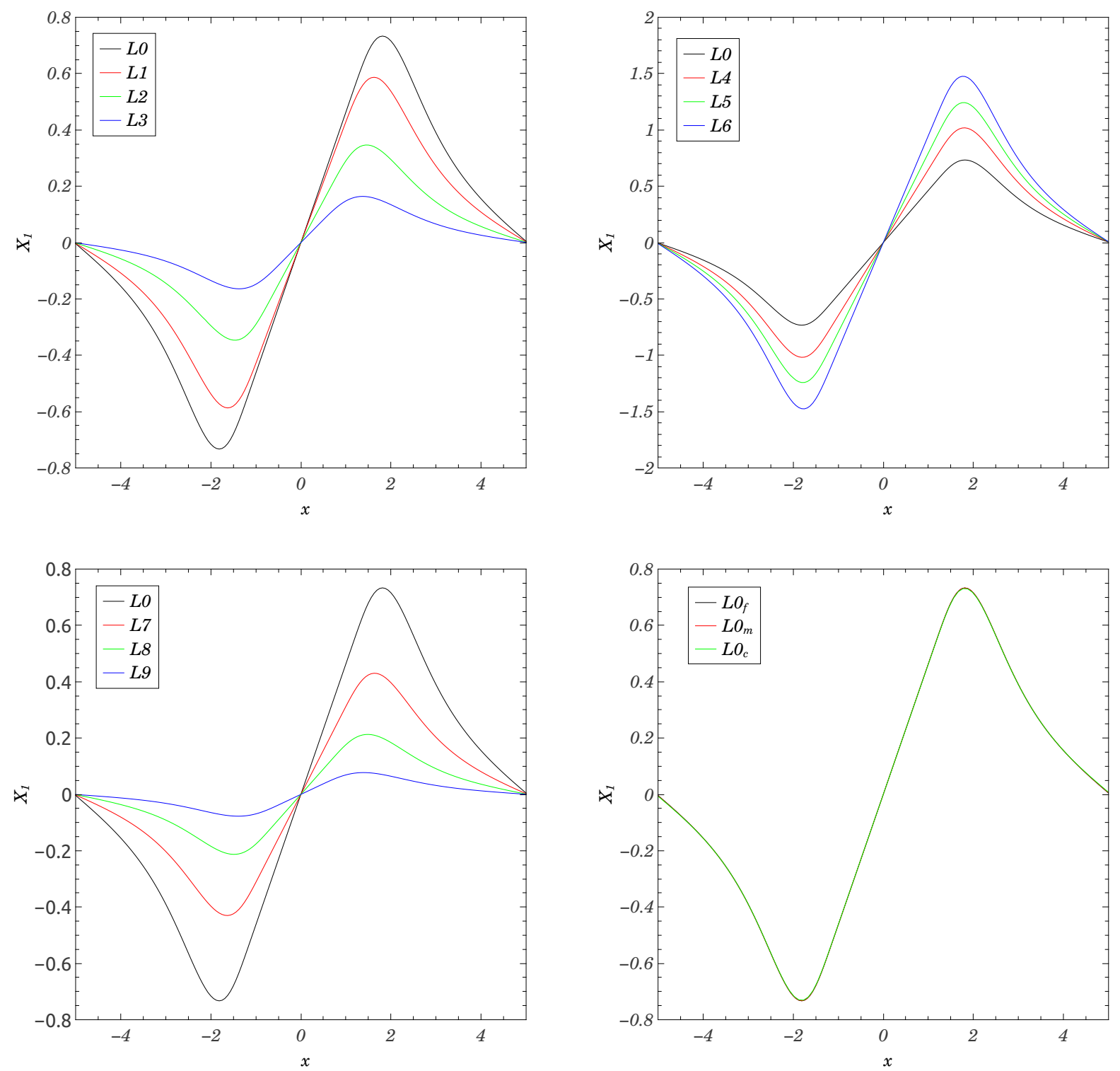

FIG. 2: $X_{1}$ of PBHLs as a function of position.

\section{Theoretical estimation}

The total energy radiated by one PBH in an expanding PBHL between $\tau$ and $\tau+\Delta \tau$ can be considered as the gravitational radiation from an accelerated mass estimated by [26]

$$
\begin{aligned}
E & =\frac{1}{2 \pi^{2}} \int d \Omega \Lambda_{i j, k l}(\hat{\mathbf{n}}) \int_{0}^{\infty} d \omega \omega^{2} \tilde{T}_{i j}(\omega, \omega \hat{\mathbf{n}}) \tilde{T}_{k l}^{*}(\omega, \omega \hat{\mathbf{n}}) \\
& \approx \frac{\gamma^{2}(\tau) m_{\mathrm{PBH}}^{2}}{2 \pi} T[v(\tau)+v(\tau+\Delta \tau)]^{2}[v(\tau)-v(\tau+\Delta \tau)]^{2} \int d \Omega \frac{\sin ^{4} \theta}{[1-v(\tau) \cos \theta]^{2}}
\end{aligned}
$$

where the energy-momentum tensor is

$$
\tilde{T}^{i j}(\omega, \omega \hat{\mathbf{n}})=2 \pi \delta\left(\omega-\omega_{0}\right) \frac{-i m_{\mathrm{PBH}}}{\omega}\left[\frac{\gamma(\tau) v^{i}(\tau) v^{j}(\tau)}{1-v(\tau) \cos \theta}-\frac{\gamma(\tau+\Delta \tau) v^{i}(\tau+\Delta \tau) v^{j}(\tau+\Delta \tau)}{1-v(\tau+\Delta \tau) \cos \theta}\right],
$$



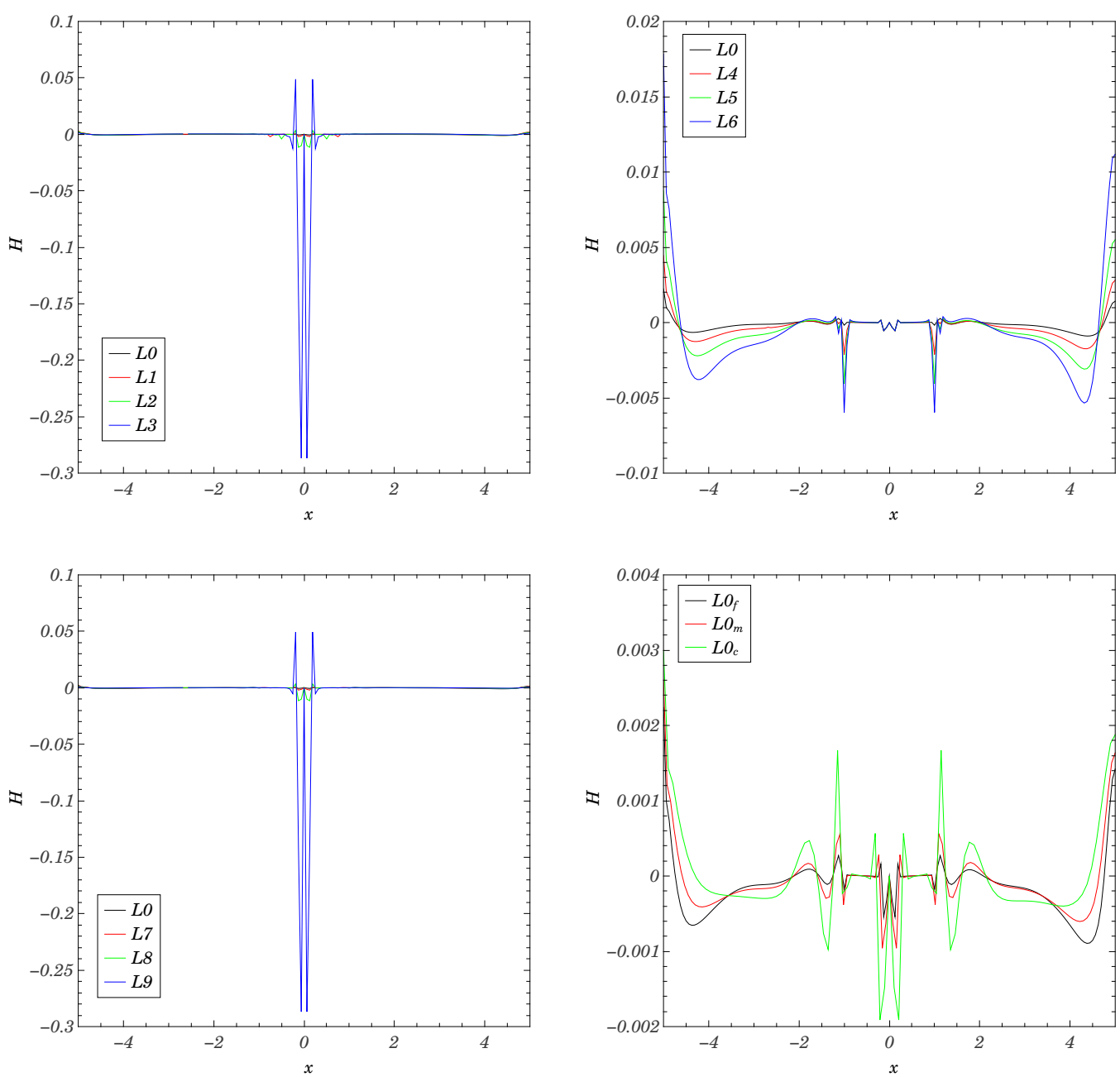

FIG. 3: The initial Hamiltonian constraint violation of PBHLs.

the Lambda tensor $\Lambda_{i j, k l}(\hat{\mathbf{n}})$ is

$$
\Lambda_{i j, k l}(\hat{\mathbf{n}})=\delta_{i k} \delta_{j k}-\frac{1}{2} \delta_{i j} \delta_{k l}-n_{j} n_{l} \delta_{i k}-n_{i} n_{k} \delta_{j l}+\frac{1}{2} n_{k} n_{l} \delta_{i j}+\frac{1}{2} n_{i} n_{j} \delta_{k l}+\frac{1}{2} n_{i} n_{j} n_{k} n_{l},
$$

the scalar product of the direction of gravitational radiation $\hat{\mathbf{n}}$ and the velocity of $\mathrm{PBH} \mathbf{v}$ is $n_{i} v^{i}=v \cos \theta, \omega_{0}$ is the frequency of the gravitational radiation at $\tau, T=2 \pi \delta(0) \approx \Delta \tau$ and $\gamma=\left(1-v^{2}\right)^{-1 / 2}$. The term of $[v(\tau)-v(\tau+\Delta \tau)]^{2}$ predicts that, in our PBHLs, the total energy radiated when $\tau \lesssim 14$ is larger than that when $\tau \gtrsim 14$ because $\left|D_{\text {edge }}(\tau \lesssim 14)^{\prime \prime}\right|$ is much larger than $\left|D_{\text {edge }}(\tau \gtrsim 14)^{\prime \prime}\right|$ as show in Fig. 4. The term of $[v(\tau)+v(\tau+\Delta \tau)]^{2}$ predicts that when $\tau \lesssim 14$ the total energy radiated in L4 should be larger than that in L0 and L7 because $D_{\text {edge }}(\tau)^{\prime}$ of L4 is larger than that of L0 and L7. Similarly, according to the initial distribution of $K=K_{c} W(r)$, the total energy radiated in PBHLs should not be uniform and the locations far away from the center are full of more radiation energy.

\section{Simulation results}

Fig. 6 shows the waveforms of GWs, as estimated by the Newman-Penrose scalar $\Psi_{4}(\tau)=h_{+}^{\prime \prime}-i h_{\times}^{\prime \prime}$, produced at several distances $r$ in expanding PBHLs with different $\rho_{t}$. For $\tau \lesssim 14$, there is an obvious production of GWs and we also find an obvious deceleration in Fig. 4, for $\tau \gtrsim 14$, the amplitude of waveforms decreases and there is a plateau for $D_{\text {edge }}(\tau)^{\prime}$ in Fig. 4. That is to say, there are some gravitational potential energy among PBHs converted to the GWs radiation and $\operatorname{Re}\left[\Psi_{4}^{2,0}\right] \propto\left|D_{\text {edge }}(\tau)^{\prime \prime}\right|$. The amplitude of waveforms increases with $\rho_{t}$ increasing, which 

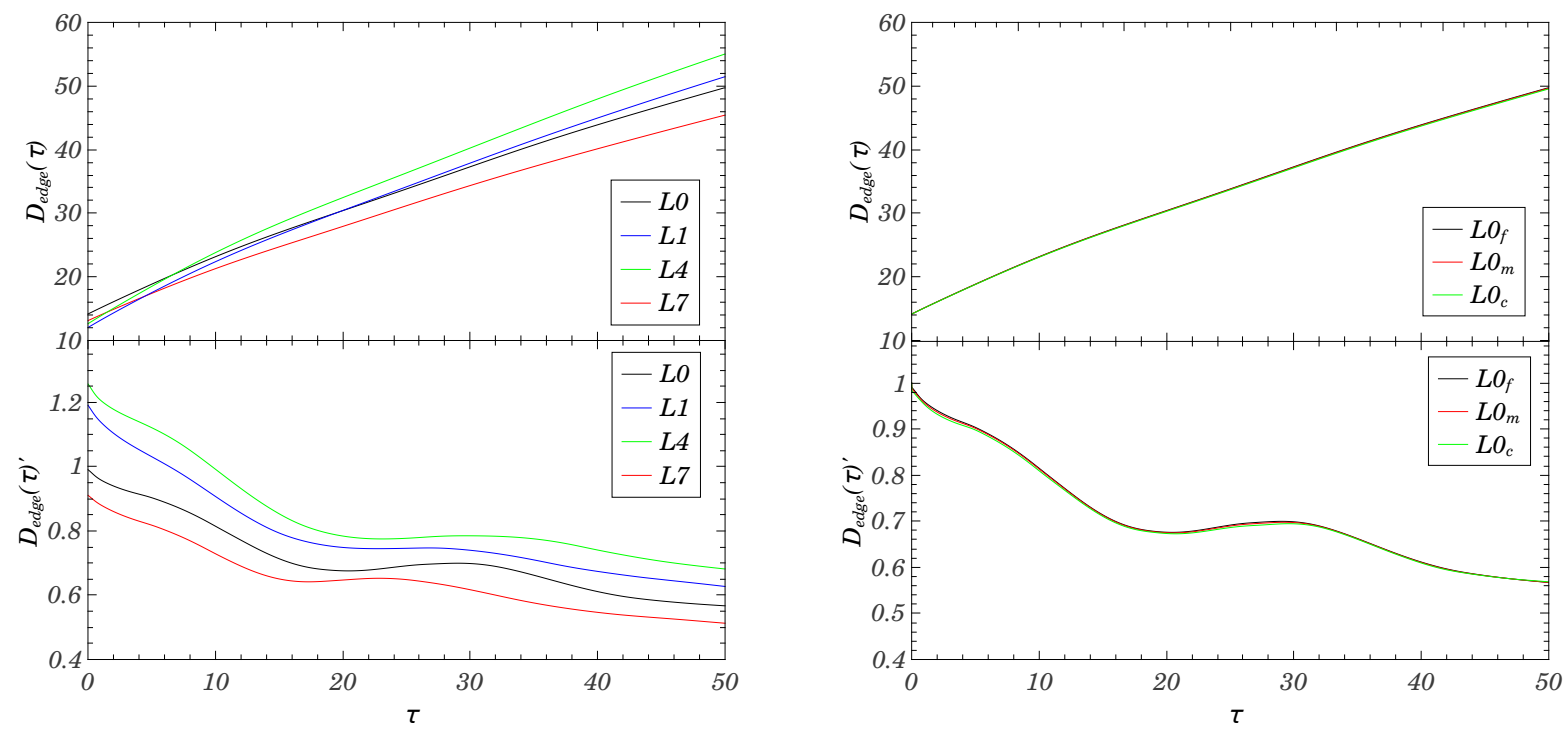

FIG. 4: Expansion of four PBHLs with the smallest initial Hamiltonian constraint violation in each group.
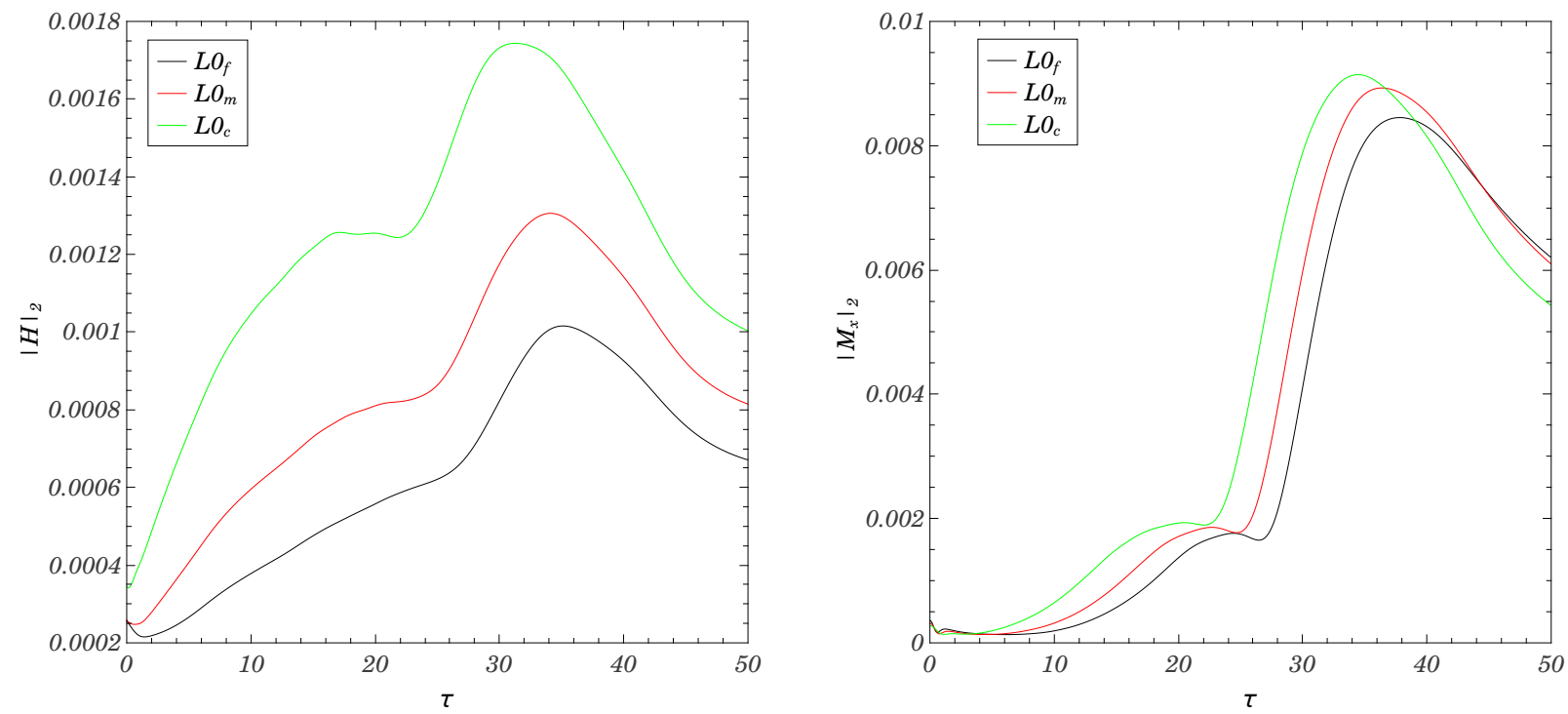

FIG. 5: $L_{2}$ norms of the Hamiltonian and momentum constraint for $L 0$ with spacing of the finest one of 5 refinement levels equal to $0.0625,0.078125$ and 0.1041667 respectively.

also means $\operatorname{Re}\left[\Psi_{4}^{2,0}\right] \propto D_{\text {edge }}(\tau)^{\prime}$ since the $D_{\text {edge }}(\tau)^{\prime \prime}$ is not sensitive to $\rho_{t}$ as shown in Fig. 4 . The amplitude of GWs increases with $r$ increasing, which means the expansion of PBHLs is not uniform and the initial distribution of $K=K_{c} W(r)$ keeps a faster expansion at positions far away from PBHs. All of above features are consistent with theoretical predictions.

The right plots in Fig. 6 show the waveforms of GWs produced at several distances $r$ in $L 0$. The oscillations due to numerical error at $\tau \approx 35$ in $L 0_{c}$ disappear in $L 0_{f}$, which guarantees the other oscillations in Fig. 6 are the waveforms of GWs produced in expanding PBHLs. 

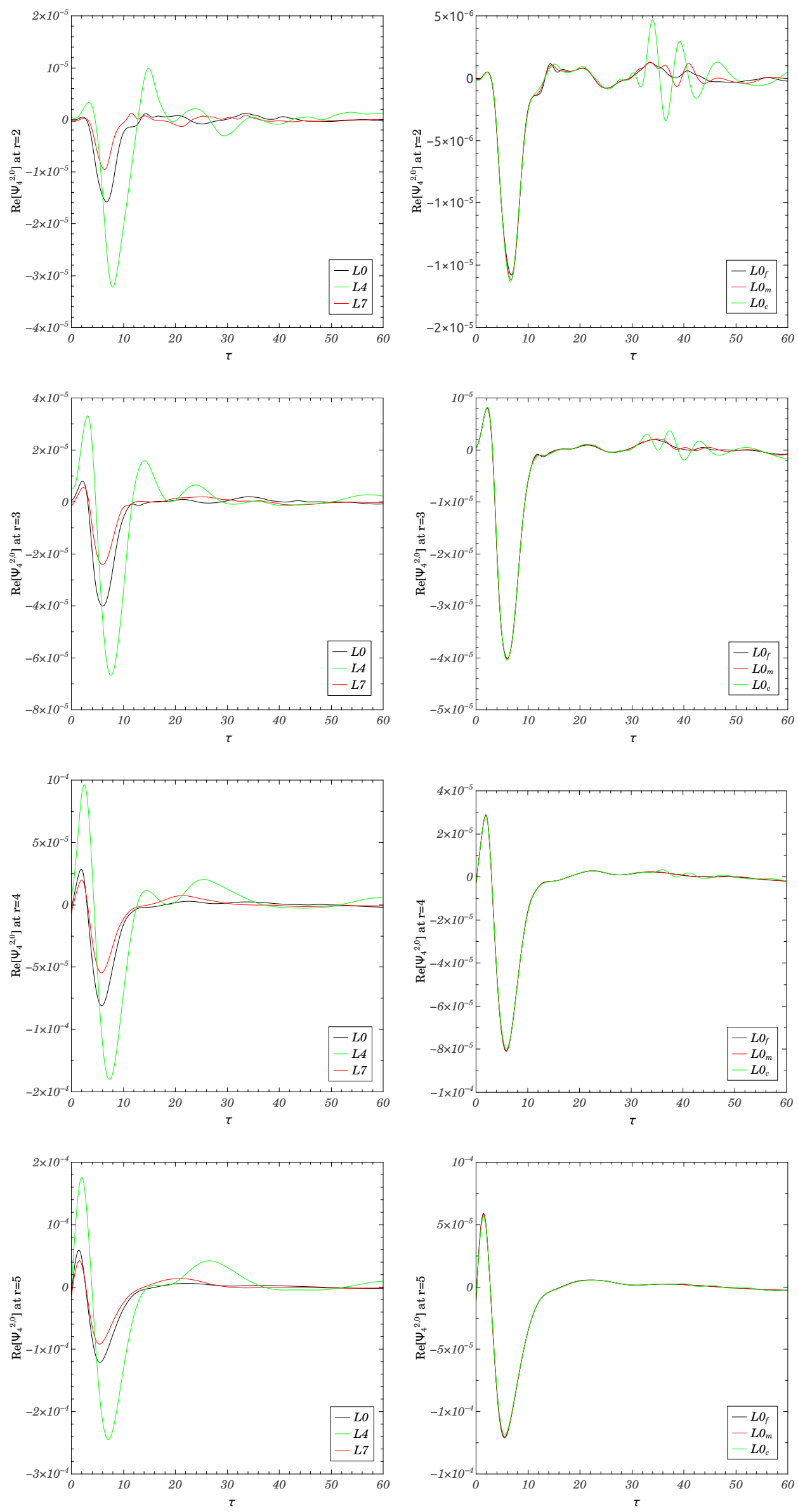

FIG. 6: A sample of GWs emitted in expanding PBHLs, as estimated by the Newman-Penrose scalar $\Psi_{4}$. 


\section{SUMMARY AND DISCUSSION}

In this paper, we first use the wide-used Einstein Toolkit to solve the Einstein constraints of PBHLs with different value of $m_{\mathrm{PBH}}$ and $f_{\mathrm{PBH}}$ which is determined by the surrounding flat-distributed dust with energy density $\rho_{m}$. From the solutions, we find that a smaller PBH mass $m_{\mathrm{PBH}}$ gives a shorter initial proper cubic edge $D_{\text {edge }}(\tau=0)$ and leads a lower initial expansion rate $-K_{c}$; a higher matter energy density $\rho_{m}$ has a higher initial expansion rate $-K_{c}$ but produces a shorter initial proper cubic edge $D_{\text {edge }}(\tau=0)$; a larger PBH mass $m_{\mathrm{PBH}}$ and a smaller matter energy density $\rho_{m}$ still produce a larger initial proper cubic edge $D_{\text {edge }}(\tau=0)$ but lead a lower initial expansion rate $-K_{c}$; a smaller $\mathrm{PBH}$ mass $m_{\mathrm{PBH}}$ violates the Hamiltonian constraint more severely; the $\mathrm{PBH}$ with larger mass will be gravitationally coupled to its neighbors more tightly; the matter surrounding the PBH suppresses the gravitational correlation among PBHs.

And then we simulate the expansion of PBHLs with the smallest initial Hamiltonian constraint violation in each group. From the evolutions, we find that $f_{\mathrm{PBH}}$ plays an important role during the evolution of PBHLs; the PBHL with a larger $\rho_{t}$ no matter due to an extra $\rho_{m}$ or a larger $m_{\mathrm{PBH}}$ expands faster; a PBHL with a larger $-K_{c}$ will keep expanding faster for ever; the motion of PBHs caused by the expansion of PBHLs does occur at speeds close to that of light. Meanwhile, we use both analytical estimates and numerical simulations to cross check the production of GWs in expanding PBHLs and find that $\operatorname{Re}\left[\Psi_{4}^{2,0}\right] \propto D_{\text {edge }}(\tau)^{\prime} D_{\text {edge }}(\tau)^{\prime \prime}$.

Comparing with the black hole and neutron star binaries' coalescence, there is an abnormal feature that the amplitude of GWs doesn't decrease with radius but increase with radius in expanding PBHLs. Our explanation is that the final waveform of GWs at any location is the superposition of eight waveforms of GWs produced by eight adjacent PBHs in expanding PBHLs and every waveform of GWs obeys the theoretical estimation (13.

There is one caveat: the total energy density $\rho_{t}$ of the real Universe in matter dominated era is much smaller than our exaggerated initial values $\sim 10^{-3}$. As we known, in SI units, this value corresponds to $6.1727 \cdot 10^{17} \mathrm{~kg} / \mathrm{m}^{3}$ hence $H^{2}=3.45 \cdot 10^{47} \mathrm{~km}^{2} / \mathrm{s}^{2} / \mathrm{Mpc}^{2}$. Although we can suppress $\rho_{t}$ through enlarging the volume of our cubic until a reasonable $\rho_{t}$ compared to the real Universe in dominated matter era, we can't afford the accompanying high computational cost. Therefore, we wouldn't give the forecast whether GWs detectors can detect such signals presented in our paper or not in future.

Acknowledgments We would like to thank Xiao Guo and You-Jun Lu for their helpful discussions and advices on this paper. This work is partly supported by the National Natural Science Foundation of China under grant No. 11690024, the Strategic Priority Program of the Chinese Academy of Sciences (Grant No. XDB 23040100).

[1] B. P. Abbott et al. [LIGO Scientific and Virgo Collaborations], "Observation of Gravitational Waves from a Binary Black Hole Merger," Phys. Rev. Lett. 116, no. 6, 061102 (2016) doi:10.1103/PhysRevLett.116.061102 arXiv:1602.03837 [gr-qc]].

[2] B. P. Abbott et al. [LIGO Scientific and Virgo Collaborations], "GW151226: Observation of Gravitational Waves from a 22-Solar-Mass Binary Black Hole Coalescence," Phys. Rev. Lett. 116, no. 24, 241103 (2016) doi:10.1103/PhysRevLett.116.241103 arXiv:1606.04855 [gr-qc]].

[3] B. P. Abbott et al. [LIGO Scientific and VIRGO Collaborations], "GW170104: Observation of a 50-Solar-Mass Binary Black Hole Coalescence at Redshift 0.2," Phys. Rev. Lett. 118, no. 22, 221101 (2017) Erratum: [Phys. Rev. Lett. 121, no. 12, 129901 (2018)] doi:10.1103/PhysRevLett.118.221101, 10.1103/PhysRevLett.121.129901 arXiv:1706.01812 [gr-qc]].

[4] B. P. Abbott et al. [LIGO Scientific and Virgo Collaborations], "GW170814: A Three-Detector Observation of Gravitational Waves from a Binary Black Hole Coalescence," Phys. Rev. Lett. 119, no. 14, 141101 (2017) doi:10.1103/PhysRevLett.119.141101 arXiv:1709.09660 [gr-qc]].

[5] B. P. Abbott et al. [LIGO Scientific and Virgo Collaborations], "GW170817: Observation of Gravitational Waves from a Binary Neutron Star Inspiral," Phys. Rev. Lett. 119, no. 16, 161101 (2017) doi:10.1103/PhysRevLett.119.161101 arXiv:1710.05832 [gr-qc]].

[6] P. A. R. Ade et al. [Planck Collaboration], "Planck 2015 results. XIII. Cosmological parameters," Astron. Astrophys. 594, A13 (2016) doi:10.1051/0004-6361/201525830 arXiv:1502.01589 [astro-ph.CO]].

[7] J. Garcia-Bellido, D. G. Figueroa and A. Sastre, "A Gravitational Wave Background from Reheating after Hybrid Inflation," Phys. Rev. D 77, 043517 (2008) doi:10.1103/PhysRevD.77.043517 arXiv:0707.0839 [hep-ph]].

[8] M. Maggiore, "Gravitational wave experiments and early universe cosmology," Phys. Rept. 331, 283 (2000) doi:10.1016/S0370-1573(99)00102-7 gr-qc/9909001.

[9] R. W. Lindquist and J. A. Wheeler, "Dynamics of a Lattice Universe by the Schwarzschild-Cell Method," Rev. Mod. Phys. 29, 432 (1957).

[10] C. M. Yoo, H. Abe, K. i. Nakao and Y. Takamori, "Black Hole Universe: Construction and Analysis of Initial Data," Phys. Rev. D 86, 044027 (2012) doi:10.1103/PhysRevD.86.044027 arXiv:1204.2411 [gr-qc]]. 
[11] C. M. Yoo, H. Okawa and K. i. Nakao, Phys. Rev. Lett. 111, 161102 (2013) doi:10.1103/PhysRevLett.111.161102 arXiv:1306.1389 [gr-qc]].

[12] E. Bentivegna and M. Korzynski, "Evolution of a family of expanding cubic black-hole lattices in numerical relativity," Class. Quant. Grav. 30, 235008 (2013) doi:10.1088/0264-9381/30/23/235008 arXiv:1306.4055 [gr-qc]].

[13] E. Bentivegna, T. Clifton, J. Durk, M. Korzyski and K. Rosquist, "Black-Hole Lattices as Cosmological Models," Class. Quant. Grav. 35, no. 17, 175004 (2018) doi:10.1088/1361-6382/aac846 arXiv:1801.01083 [gr-qc]].

[14] F. Loffler et al., "The Einstein Toolkit: A Community Computational Infrastructure for Relativistic Astrophysics," Class. Quant. Grav. 29, 115001 (2012) doi:10.1088/0264-9381/29/11/115001 arXiv:1111.3344 [gr-qc]].

[15] E. Bentivegna, "Solving the Einstein constraints in periodic spaces with a multigrid approach," Class. Quant. Grav. 31, 035004 (2014) doi:10.1088/0264-9381/31/3/035004 arXiv:1305.5576 [gr-qc]].

[16] J. D. Brown, P. Diener, O. Sarbach, E. Schnetter and M. Tiglio, "Turduckening black holes: An Analytical and computational study," Phys. Rev. D 79, 044023 (2009) doi:10.1103/PhysRevD.79.044023 arXiv:0809.3533 [gr-qc]].

[17] C. Reisswig, C. D. Ott, U. Sperhake and E. Schnetter, "Gravitational Wave Extraction in Simulations of Rotating Stellar Core Collapse," Phys. Rev. D 83, 064008 (2011) doi:10.1103/PhysRevD.83.064008 arXiv:1012.0595 [gr-qc]].

[18] McLachlan, a public BSSN code URL. http://www.cct.lsu.edu/ eschnett/McLachlan/

[19] T. W. Baumgarte and S. L. Shapiro, "On the numerical integration of Einstein's field equations," Phys. Rev. D 59, 024007 (1999) doi:10.1103/PhysRevD.59.024007 gr-qc/9810065.

[20] M. Shibata and T. Nakamura, "Evolution of three-dimensional gravitational waves: Harmonic slicing case," Phys. Rev. D 52, 5428 (1995). doi:10.1103/PhysRevD.52.5428

[21] M. Alcubierre et al., "Towards a stable numerical evolution of strongly gravitating systems in general relativity: The Conformal treatments," Phys. Rev. D 62, 044034 (2000) doi:10.1103/PhysRevD.62.044034 gr-qc/0003071.

[22] P. Msta et al., "GRHydro: A new open source general-relativistic magnetohydrodynamics code for the Einstein Toolkit," Class. Quant. Grav. 31, 015005 (2014) doi:10.1088/0264-9381/31/1/015005 [arXiv:1304.5544 [gr-qc]].

[23] L. Baiotti, I. Hawke, P. J. Montero, F. Loffler, L. Rezzolla, N. Stergioulas, J. A. Font and E. Seidel, "Three-dimensional relativistic simulations of rotating neutron star collapse to a Kerr black hole," Phys. Rev. D 71, 024035 (2005) doi:10.1103/PhysRevD.71.024035 gr-qc/0403029.

[24] I. Hawke, F. Loffler and A. Nerozzi, "Excision methods for high resolution shock capturing schemes applied to general relativistic hydrodynamics," Phys. Rev. D 71, 104006 (2005) doi:10.1103/PhysRevD.71.104006 [gr-qc/0501054].

[25] S. Brandt and B. Bruegmann, Phys. Rev. Lett. 78, 3606 (1997) doi:10.1103/PhysRevLett.78.3606 gr-qc/9703066].

[26] M. Maggiore, "Gravitational Waves. Vol. 1: Theory and Experiments," 